\title{
STUDY OF HEAT TRANSFER AFFECTED BY RADIATION IN A TWO DIMENSIONAL ENCLOSURE
}

\author{
Prerana Nashine ${ }^{1}$, A. K. Satapathy ${ }^{2}$ \\ 1,2 Department of Mechanical Engineering, National Institute of Technology ,Rourkela, India \\ Email: 1prerana.mech08@gmail.com
}

Abstract -

The calculation of radiative transfer in a two dimensional enclosure with participating media is based on the Finite volume method. Radiative heat transfer in a black square enclosure with a heated bottom wall in an absorbing, emitting and gray gas medium has been studied. Attention is directed towards the evaluation of radiative incident radiation energy effect with different values of time when energy transport is characterized as a radiant phenomenon. The spatial and angular discretization of two-dimensional geometry is based on finite volume method. The effect of incident radiation energy goes on increasing with time has been concluded by the present study.

Key words: Radiative transfer, Two-dimensional, Heat transfer, Finite volume method, Incident radiation

\section{INTRODUCTION}

Few illustrations in which the transient nature of thermal radiation has to be considered are pulsed laser interactions with materials, micro-scale systems, laserinduced shock waves, optical tomography, laser therapy, remote detecting of turbid media of weather and sea. The radiation $\mathrm{n}$ heat transfer is apparent in various engineering applications for semi transparent materials STM such as the glass industry, molten salt media, brous materials, infrared heating as well as the utilization of solar energy, etc. Since the spatial domain is divided into a finite number of control volumes in the FVM, this method has a computational interface with other controlvolume based CFD approach.

\section{LITERATURE REVIEW}

Trivedi et.al.(2005) used the short pulse laser for minimally invasive detection scheme has become an indispensable tool in the technological arsenal of modern medicine and biomedical engineering. Mishra et.al.(2006) proposed a formulation of the collapsed dimension method (CDM) which is similar to the discrete ordinate method (DOM) . Hahn et.al. (1997) proposed a model for the calculation of transient combined radiativeconductive heat transfer in heterogeneous semitransparent materials at elevated temperatures. Guo and kumar (2001) presented the discrete-ordinates method which is formulated to solve transient radiative transfer with the incorporation of a transient term in the transfer equation in two-dimensional rectangular enclosures. Early studies of this subject were reviewed in detail by Viskanta and Anderson .It has attracted further research in recent years as to the combined heat transfer with multi-dimension Siegel.(1992) under transient state or with refractive index greater than unity in a scattering medium. Among others, there is the finite volume method (FVM) for radiation by Chai. etal.(1994) and Chui.etal. (1993) which has been successfully applied to several problems of body-fitted geometries as given by Baek.etal. (1998).

\section{MATHEMATICAL EQUATIONS}

A two dimensional radiative transfer equation is given by

$$
\begin{aligned}
& \frac{1}{c} \frac{d I\left(r, \hat{s}^{\prime}\right)}{d t}+\frac{d I(r, \hat{s})}{d s} \\
& =-\beta(r) I(r, \hat{s})+S(r, \hat{s})
\end{aligned}
$$

The modified extinction coefficient $\beta_{\mathrm{m}}$ and $\mathrm{Sm}$ are given by

$$
\begin{gathered}
\beta_{m}^{l}=\kappa+\sigma-\frac{\sigma}{4 \pi} \phi^{l l} \Delta \Omega^{l} \\
S_{m}^{l}=\kappa I_{b}+\frac{\sigma}{4 \pi} \sum_{l^{\prime}=1, l^{\prime} \neq 1}^{M} I^{l^{\prime}} \phi^{l^{l} l} \Delta \Omega^{l^{\prime}}
\end{gathered}
$$


And the equation (1) can be written as follows

$$
\begin{aligned}
& \int_{\Delta \Omega} \int_{\Delta v} \int_{\Delta t} \frac{1}{c} \frac{\partial I}{\partial t} d t d v d \Omega+\int_{\Delta t} \int_{\Delta \Omega} \int_{\Delta v} \frac{\partial I}{\partial s} d v d \Omega d t \\
& =\iint_{\Delta t} \int_{\Delta \Omega}\left(-\beta_{\Delta v} I+S_{m}\right) d v d \Omega d t
\end{aligned}
$$

The above equation can be simplified as follows

$$
\begin{aligned}
& \frac{1}{c}\left(I_{P}-I_{P}^{0}\right) \frac{\Delta x}{\Delta t} \Delta \Omega+D_{c x}\left(I_{e}-I_{w}\right) \\
& +D_{c y}\left(I_{n}-I_{s}\right)=\left(-\beta_{m} I_{P}+S_{m}\right) \Delta v \Delta \Omega
\end{aligned}
$$

Where $I_{p}^{0}$ shows the intensity at previous time step and $I_{p}$ at present time step. Pursuing the discretization practice of Chai.et al., (1994) the second term in the left hand side of the equation and the term of right hand side have been discretized. $l_{e}$ and $I_{w}$ are the intensities at east and west control volume faces. Intensity $I_{p}$ and $I_{p} I_{p}^{0}$ are the centre node intensity at the end and at the beginning of time step respectively. To relate the boundary intensities to the nodal intensity spatial differencing scheme are needed and one such available scheme is the step scheme. A final discretization equation (for $D_{c x}>$ $0 D_{c x}>0$ ) can be reduced in a standard form of finite volume method as

$$
a_{P}^{\prime} I_{P}^{\prime}=a_{W}^{\prime} I_{P}^{\prime}+a_{S}^{\prime} I_{S}^{\prime}+b
$$

For the solution of Eq. (5) the initial intensity is prescribed according to initial boundary condition and the terms $a_{p}$ the coefficient of discretization equation and $b$ the source term in the discretization equation can be written in a discretized form as follows

$$
\begin{aligned}
& a_{w}=D_{c x} \\
& a_{P}=D_{c x}+D_{c y}+\beta_{m, p} \Delta x \Delta \Omega+\frac{1}{c} \frac{\Delta v}{\Delta t} \Delta \Omega \\
& b=D_{c x}+D_{c y}+S_{m, P} \Delta v \Delta \Omega+\frac{1}{c} I_{p}^{0} \frac{\Delta v}{\Delta t} \Delta \Omega \\
& D_{c x}=\int_{\Delta \Omega}\left(\hat{s} . \hat{n}_{x}\right) d \Omega
\end{aligned}
$$

$$
D_{c y}=\int_{\Delta \Omega}\left(\hat{s} . \hat{n}_{y}\right) d \Omega
$$

The problem of TRTE in a two dimensional slab investigated in this article using the step scheme of finite volume method technique which sets the downward nodal intensity equal to the upstream boundary intensity. The computation domain has been discretized into $100 \times 100$ uniform control volumes and $2 \times 8$ control angles with uniform theta and phi. The centre node intensity is being calculated by equation(8)

$$
I_{P}{ }^{\prime}=\frac{\Delta y D_{c y}^{\prime} I_{w}^{\prime}+\Delta x D_{c y}^{\prime} I_{s}^{\prime}+\left(S_{m}^{\prime}\right)_{P} \Delta v \Delta \Omega^{\prime}}{\Delta y D_{c x}^{\prime}+\Delta x D_{c y}^{\prime}+\left(\beta_{m}^{\prime}\right)_{P} \Delta v \Delta \Omega^{\prime}}
$$

\section{RESULTS AND DISSCUSSION}

The study of radiation has been carried out in a two dimensional square enclosure with heated bottom wall. The bottom wall is kept at a temperature of $(1 / \sigma)^{1 / 4}$ where $\sigma$ is Stefan's Boltzmann constant and all other walls are kept at absolute zero Kelvin temperature. The effect of irradiation at different time step i.e., at $t=0.6,0.8$, 1.0, 1.2 and $1.8 \mathrm{sec}$ has been shown by the contour plot as follows. The incident radiation energy is at its peak at the bottom wall and gradually goes on reducing.

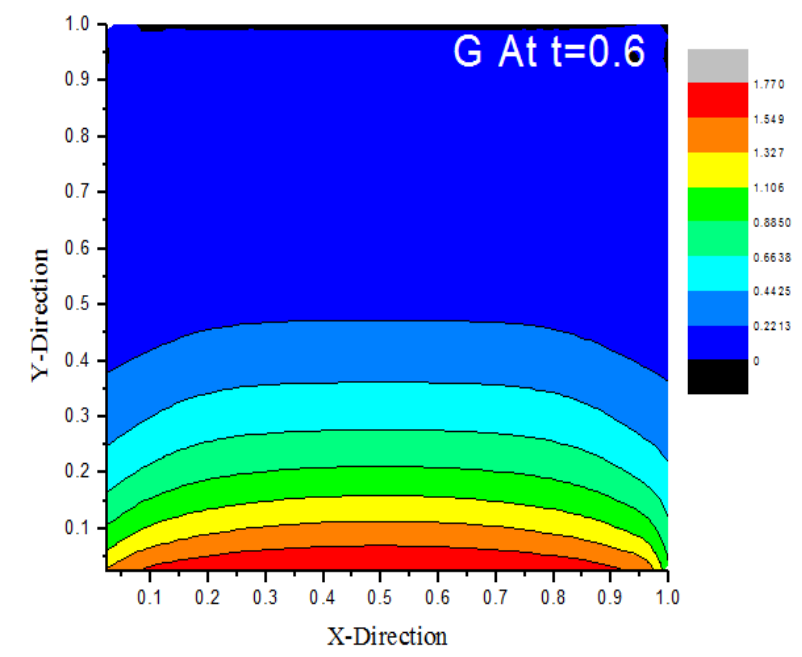

Fig. 1. Varriation of irradiation at $t=0.6$

Figure 1 shows the effect of incident radiation energy at $t=0.6$. The bottom highlighted area shows that the incident radiation energy is maximum while it goes on 
decreasing while moving towards top wall since some amount of energy gets scattered in the medium.

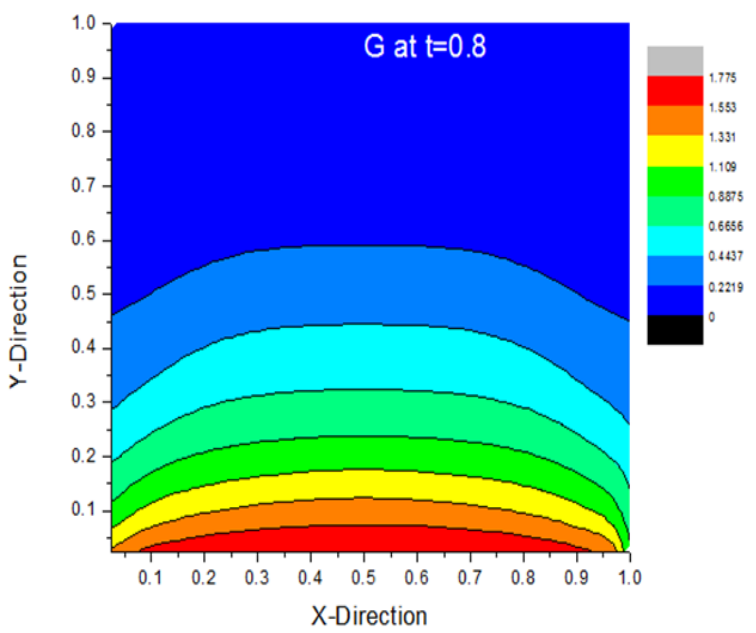

Fig.2. Varriation of irradiation at $\mathrm{t}=0.8$

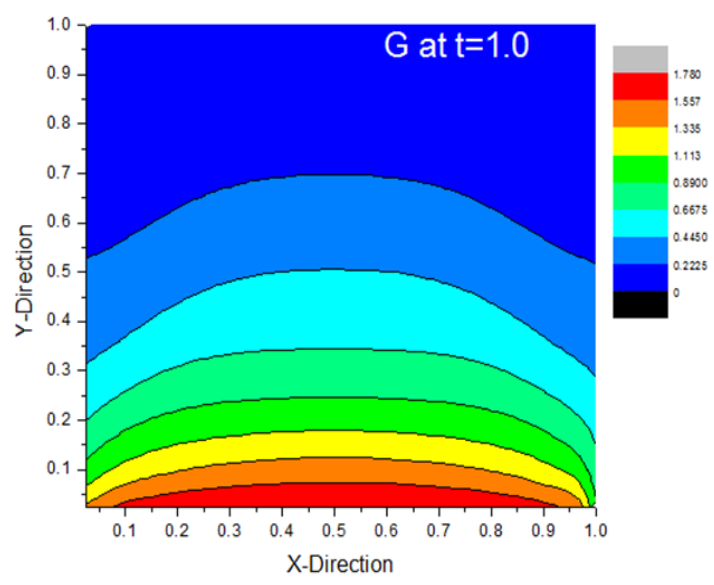

Fig.3. Varriation of irradiation at $\mathrm{t}=1.0$

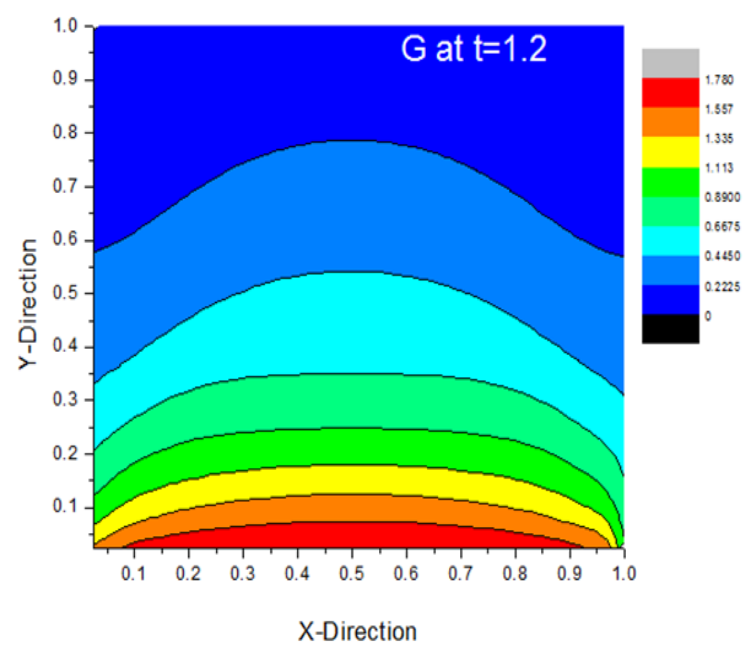

Fig.4. Varriation of irradiation at $\mathrm{t}=1.2$
Variation of incident radiation energy at time $t=0.8$ and $t=1.10$ has been depicted by the contour plot in figure 2 and 3 . At $t=0.8$ the incident radiation approaches more towards the top wall as compared to $t=0.6$ while it is less as compared incident radiation energy at $t=1.0$ seconds.

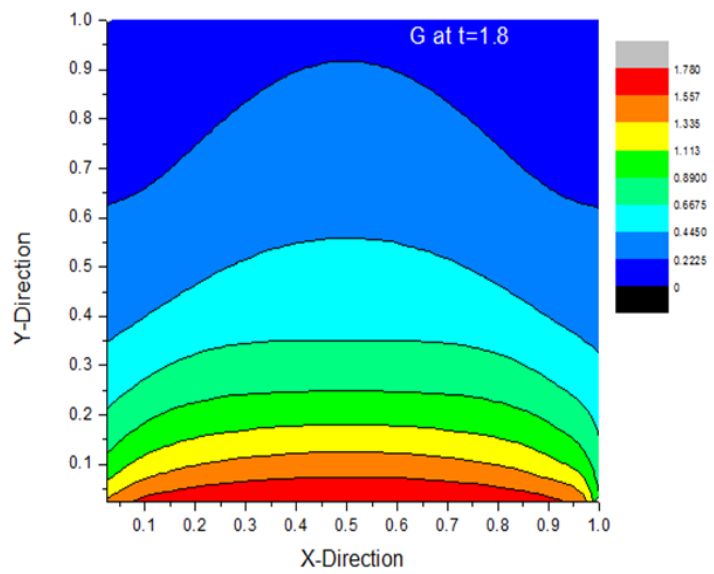

Fig.5. Varriation of irradiation at $\mathrm{t}=1.8$

Contour plot of irradiation at $t=1.2$ and $t=1.8$ has been shown in figure 3 and figure 4.Irradiation is maximum for $t=1.8$ and minimum at $t=0.6$. The radiative effect reaches up to the top wall by the time and the effect of irradiation also goes on increasing with time ,its effect is maximum for $t=1.8$ and it has its lowest value at $t=0.6$, which can be easily seen in the contour plots shown.

\section{v. CONCLUSIONS}

In this existing research, we present the results about the transient radiative temperature variation in twodimensional enclosure of square geometry. Computation was done for the construction of temperature field for a transient radiation problem. The discretized radiative transfer equation following with the computation has been done using the finite volume method. In the transient problem due to the high propagation speed of the thermal radiation, transport of thermal radiation was regarded as an instantaneous process. At any time level, with volumetric radiation known from the previous time level, equation was solved, procedure and the radiative information was acquired using the FVM. Incident radiation energy is maximum at the bottom boundary and goes on reducing as it approaches the top wall because the energy absorption by the participating medium 
reduces the radiative heat transfer. Incident energy effect is less at $t=0.3$ and the effect is maximum for $t=1.8$ and thus we conclude that the effect of irradiation increases with time.

\section{NOMENCLATURE}

\section{$\beta_{m} \quad$ Extinction coefficient}

$S_{m} \quad$ source term

$I_{p}^{0} \quad$ Intensity at previous time step

$I_{p} \quad$ Intensity at centre nodal point

$l_{e} \quad$ Intensities at east control volume faces

Iw Intensities at west control volume faces

$I_{p} \quad$ Intensity at $I_{p}^{0}$ the centre node

$1_{p}$ Intensity at the beginning of time step

$P \quad$ centre node

$w \quad$ west node

b sourse term

\section{REFERENCES}

[1] Baek,S.W., Kim, M.Y., Kim, J.S., 1998, Nonorthogonal finitevolume solutions of radiative heat transfer in a threedimensional enclosure, Numerical Heat Transfer , 34 (4),pp. 419-437.

[2] Chai,J.C, Lee,H.S., Patankar, S.V., 1994, Finite volume method for radiation heat transfer ,Journal of thermo physics and heat transfer, 8 (3),pp. 419-425.
[3] Chui, E.H., Raithby, G.D.,1993, Computation of radiant heat transfer on a nonorthogonal mesh using the finite-volume method, Numerical Heat Transfer , 23(B),pp. 269-288.

[4] Guo,Z., Kumar,S., 2000, Discrete-ordinates solution of shortpulsed laser transport in two-dimensional turbid media. Applied Optics, 40(19).

[5] Hahn,O., Raether,F., Arduini-Schuster, M. C., Fricke, J.,1997,Transient coupled conductive -radiative heat transfer in absorbing ,emitting and scattering media: application to laser-flash measurements on ceramic materials, International Journal of Heat Mass Transfer, 40 (3),pp. 689-698.

[6] Kunc,T., Lallemand,M., Saulnier,J.B., 1984, Some new developments on coupled radiative conductive heat transfer in glasses experiments and modelling, International Journal of Heat and Mass Transfer, 27 (12),pp. 2307-2592.

[7] Mishra,S.C., Kuar,N., Roy,H.K., 2006, The DOM approach to the collapsed dimension method for solving radiative transport problems with participating media, International Journal of Heat and Mass Transfer, 49, pp.30-41.

[8] Siegel.R. Molls.F.B., 1992, Finite difference solution for transient radiative cooling of a conducting semi-transparent square region, International Journal of Heat and Mass Transfer,34(10), pp. 2579-2592.

[9] Trivedi,A., Basu,S., Mitra,K., 2005, Temporal analysis of reflected optical signals for short pulse laser interaction with nonhomogeneous tissue phantoms. Journal of Quantitative Spectroscopy \& Radiative Transfer, 93,pp. 337-348.

[10] Viskanta,R., Anderson,E.E., 1975, Heat transfer in semitransparent solids, In Advances in Heat Transfer, Academic Press,New York. 